\title{
Soft Tissue Resection for Prostatectomy Simulation
}

\author{
Miguel A. Padilla Castañeda and Fernando Arámbula Cosío \\ Image and Vision Lab., \\ Centre of Applied Research and Technology (CCADET), \\ National Autonomous University of México (UNAM), \\ México, D.F., 04510 \\ \{arambula, padillac\}@aleph.cinstrum.unam.mx
}

\begin{abstract}
In this paper we present a computer model of the prostate for prostatectomy simulation. Through the modification of the 3D mesh, the model is able to simulate resections of soft tissue produced by the user with a virtual resectoscope. At each resection the model shows deformations of the tissue surrounding the resection zone. A mass-spring 3D mesh is used to model tissue deformation due to the interaction with the surgical tool. Our model is designed to be the basis of a surgery training system for Transurethral Resection of the Prostate (TURP).
\end{abstract}

\section{Introduction}

The prostate is a chestnut sized gland located next to the bladder in human males. The urethra runs from the bladder neck through the prostate to the penile urethra. A frequent condition in men above 50 years old is the benign enlargement of the prostate known as Benign Prostatic Hyperplasia (BHP), which in some cases results in significant blockage of the urinary flow. The standard surgical procedure to treat a hypertrophied prostate gland is the Transurethral Resection of the Prostate (TURP). It essentially consists of the removal of the inner lobes of the prostate in order to relieve urinary outflow obstruction.

Less invasive surgical techniques such as Transuretral Incision of the Prostate, Microwave Therapy, Transurethral Needle Ablation, and High Frequency Focused Ultrasound, have been developed to treat BPH. However TURP remains the most commonly used surgical procedure to treat BPH [1]. During a TURP the surgeon inserts a cylindrical instrument called resectoscope - which carries in its interior a cylindrical lens and the resecting element - through the urethra of the patient up to the prostate. The surgeon then removes the lobes of the prostate by resection of small tissue chips. Mastering the TURP technique requires a highly developed handeye coordination which enables the surgeon to orientate inside the prostate, using only the monocular view of the lens of the resectoscope. Current training of TURP is mainly performed by example, with residents observing a large number of procedures performed by an experienced surgeon. Eventually, each resident is allowed to perform partial procedures under the supervision of the expert. Towards the end of the training period, the resident performs a full TURP under supervision. There are few practice 
opportunities available to the residents during the current training process. Practice is limited to the resection of artificial models which can be expensive, or potatoes which are unrealistic. Computers can help to improve TURP training through realistic simulation of prostate resections.

Realistic simulation of soft tissue cutting and deformation is one of the most important research topics in surgery simulation. The purpose is to manipulate $3 \mathrm{D}$ tissue models in real time with acceptable visual quality. Bro-Nielsen [2] reported an approach for surgery simulation based on a finite element method for real time deformations which uses a technique called condensation, in order to work only with the surface mesh elements, reducing in this way the time response of the model. Unfortunately this method is too slow for the simulation of tissue cutting operations even with simple mesh modifications. Bielser and Gross [3] reported an interactive simulation technique for surgical cuts that simulates cutting operations using a virtual scalpel. The cuts in their approach are made in a line like way, and they focus on the problem of refining the mesh along the cut line to produce continuous dissections. Gomes et al. [4] reported a computer assisted system for TURP based on real time optical tracking and a geometric model of the prostate, but which does not include a prostate model that simulates the physical behaviour of soft tissue. Ballaro et al. [5] reported a simulator for prostatic resection based on a magnetic tracker and a 3D model of the prostatic urethra which simulates deformation, resection and bleeding. The capsule of the prostate is not included in the model and therefore overall shape change of the prostate is not calculated. The paper focuses on the clinical aspects of the system and little technical detail of the model construction is provided.

In this work is reported a model for TURP simulation which incorporates tissue resection and deformation. The elastic behaviour of the whole prostate gland is modelled with the mass-spring method for deformable bodies. Resections are modelled through the removal of nodes and geometrical elements from the volumetric 3D mesh. The results show that the model is able to simulate deformations and resections performed by the user. This model is intended to be used in a surgery training system for TURP.

In the next section is described the construction of the geometric model of the prostate. In section 3 is described the use of the mass-spring method for simulation of deformations of the prostate model. In section 4 we report the tissue resection mechanism used for simulation. In section 5 we show a sequence of simulated prostate resections, and in section 6 we present the conclusions of the work reported.

\section{Geometric Model of the Prostate}

To reconstruct the three-dimensional shape of the prostate, the mesh generation method uses a set of transurethral, ultrasound images, separated by intervals of $5 \mathrm{~mm}$. To acquire the images a lead screw mechanism, rigidly fixed to the operating table, was used to move the ultrasound probe along the urethra from the bladder neck to the verumontanum (Fig. 1). 


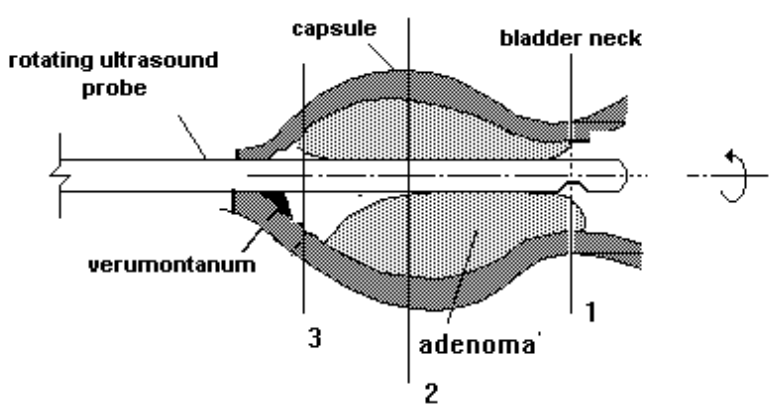

Fig. 1. Transurethral ultrasound scanning of the prostate

The lead screw mechanism maintains all the images parallel to each other and mostly transverse to the prostate. Some small distortion of the final prostate shape is likely to occur since the urethra has a natural slight bent at the middle and the ultrasound probe straightens it during image acquisition [6]. For a training simulator a slight distortion of the prostate model does not affect the usefulness of the system, as long as the final model still looks like a realistic prostate to a specialist. All the images acquired from a prostate are annotated by an expert in ultrasound (Fig. 2).

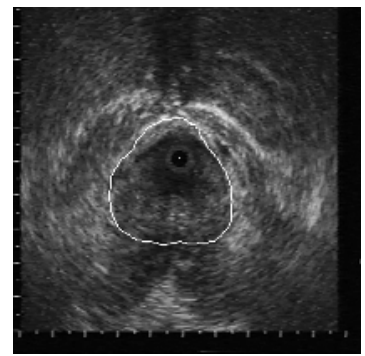

(a)

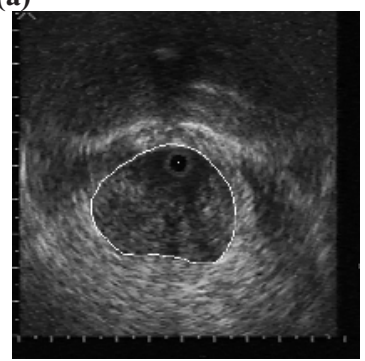

(d)

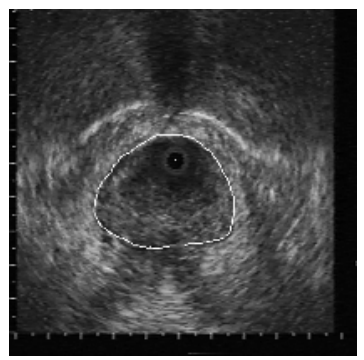

(b)

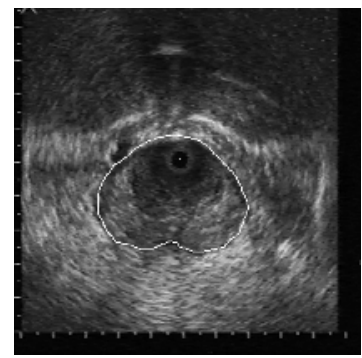

(c)

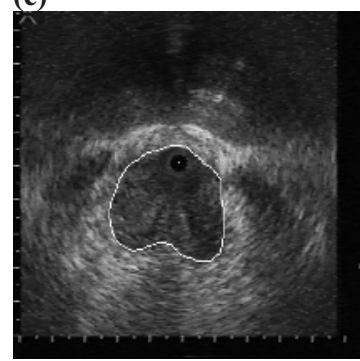

(e)

Fig. 2. Transurethral ultrasound images with the prostate contours annotated by an expert sonographer: (a) At $5 \mathrm{~mm}$ from the bladder neck; (b) At $10 \mathrm{~mm}$; (c) At $15 \mathrm{~mm}$; (d) At $20 \mathrm{~mm}$; (e) At $25 \mathrm{~mm}$

Each of the contours of the prostate capsule was sampled in a radial manner, taking as the origin the centre of the transurethral ultrasound transducer. The number 
of samples is determined by the size of the sampling angle $\alpha$, which is the control parameter of the mesh generation method. The same procedure is applied to the contour of the prostatic urethra, forming a set of cross-sections $\boldsymbol{C}$ with both capsule and urethra samples. Since it is not possible to identify the prostatic urethra from ultrasound images, we drew an approximate urethra contour on each of the ultrasound images. In this manner, the sampled points of every cross-section $\mathbf{c s}_{\mathbf{i}}$ (with the capsule and the urethra contours) in $\boldsymbol{C}$, represent the control points of the prostate shape.

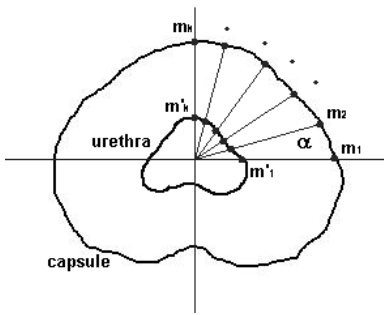

(a)

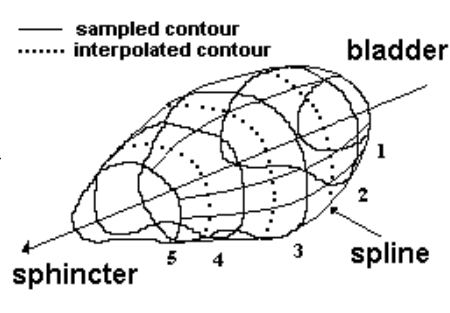

(b)

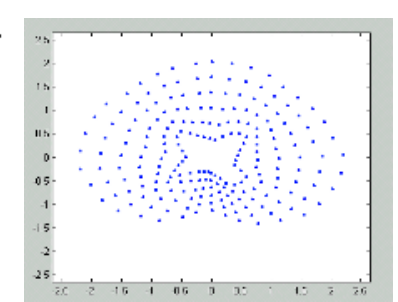

(c)

Fig. 3. (a) Radial sampling of a prostate cross-section with sampling angle $\alpha$ and $n$ sample pairs $(n=360 / \alpha)$. (b) 3D surface mesh interpolated from the transurethral ultrasound cross-sections. (c) Internal points obtained from the capsule to the urethra with $\alpha=10$ and $l=0.187828$

In order to control the uniformity of the mesh, we calculated the average length $(l)$ of all the segments $\mathbf{m}_{\mathrm{ij}}$ and $\mathbf{m}_{\mathbf{i j}}$ in $\boldsymbol{C}$, that join the control points $\mathbf{m}_{\mathbf{i}}$ and $\mathbf{m}_{\mathbf{j}}$ of the capsule, and the control point $\mathbf{m}_{\mathbf{i}}$ and $\mathbf{m}_{\mathbf{j}}$ of the urethra, respectively (Fig. 3.a). The next step is to transform the prostate shape $\boldsymbol{C}$, typically composed by 5 to 12 crosssection images (separated at $5 \mathrm{~mm}$ ), into the new shape $C^{*}$ now composed by $n$ target cross-sections separated by the average distance $l$, previously calculated. $C^{*}$ is generated using cubic spline interpolation over the control points of $\boldsymbol{C}$ (Fig. 3.b). To model the prostate as a solid body, the algorithm also interpolates, for every crosssection $\mathbf{c s}_{\mathbf{j}}$ in $\boldsymbol{C}^{*}, k$ internal sampled contours from the capsule to the urethra (Fig. 3.c). Again, the number of $k$ inner contours depends on the $l$ value. Finally, the last step is to arrange the solid body of the prostate as a mass-spring mesh of lattice form, where every node $v_{i, j, k}$ in the body is linked with at most 6 adjacent nodes $\left(v_{i+1, j, k}\right.$ $v_{i, j+l, k}, v_{i, j, k+1}, v_{i+l, j+l, k}, v_{i+1, j, k+1}$ and $\left.v_{i+1, j+1, k+1}\right)$.

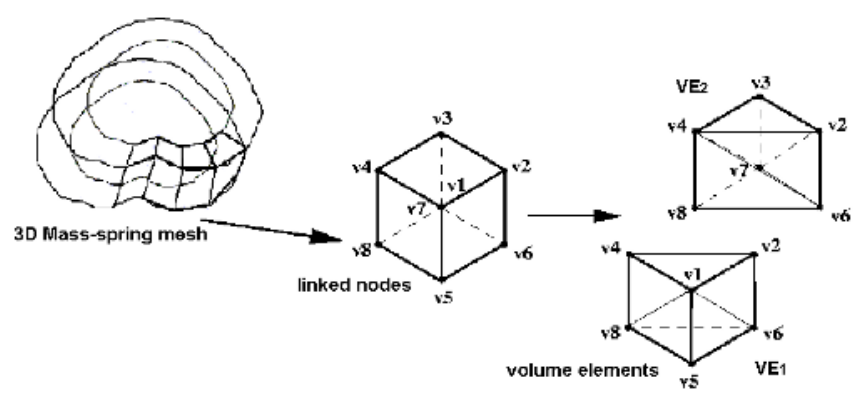

Fig. 4. Volume elements of the 3D mass-spring mesh of the prostate 
Figure 4 illustrates the arrangement of the 3D prostate mesh. Additional geometric volume elements of the form of a pentahedron $\left(V E_{1}\right.$ and $\left.V E_{2}\right)$ are constructed. As we will show in the next section, the form of these volume elements is useful for simulating tissue resection operations.

\section{Physical Model of the Prostate}

The behaviour of the prostate is modelled as a deformable body with physical characteristics like mass, stiffness and damping coefficients. These physical characteristics were adapted to the volumetric 3D mesh constructed before, to produce a viscoelastic 3D mesh. This was done using the spring-mass method [7, 8], where every node in the mesh represents a mass point that is interconnected with its neighbours by springs and which moves in a viscous medium, this method allows us to simulate real time deformations.

The dynamic behaviour of the system, formed by the spring-mass elements in the volumetric mesh, is based on the Lagrange equation of motion.

$$
m_{i} \frac{d^{2} \mathbf{x}_{\mathbf{i}}}{d t^{2}}+\gamma_{i} \frac{d \mathbf{x}_{\mathbf{i}}}{d t}+\mathbf{g}_{\mathbf{i}}\left(t, \mathbf{x}_{\mathbf{i}}\right)=\mathbf{f}_{i}\left(t, \mathbf{x}_{\mathbf{i}}\right)
$$

Where $m_{i}$ is the mass of the node $i$ in the mesh, at Cartesian coordinates $\mathbf{x}_{i} ; \gamma_{i}$ is the damping coefficient of the node (viscosity of the medium); $\mathbf{g}_{\mathbf{i}}$ is the internal elastic force and $\mathbf{f}_{\mathbf{i}}$ are the external forces acting on the node.

In this approach the internal elastic forces acting on the node $i$ are given by the following linear equation:

$$
g_{i}=\sum_{j \in N(i)} \mu_{i, j} \frac{\left(\left\|x_{i}-x_{j}\right\|-l_{i, j}^{0}\right)\left(x_{i}-x_{j}\right)}{\left\|x_{i}-x_{j}\right\|}
$$

Where $N(i)$ is the set of neighbours of the node $i ; \mu_{i, j}$ is the stiffness coefficient of the spring connecting the nodes $i$ and $j$ for every $j$ in $N(i)$; and $l_{i, j}^{0}$ is the spring length at rest position. In this manner, the deformations occur as a result of the inner elastic energy change, produced by the spring deformation. Due to the computational speed needed and for simplicity of programming, we have used the Newton-Euler integration method to solve the dynamic systems equation (1).

\section{Tissue Resection}

During a TURP the basic tissue removal mechanisms consist of the resection (using a resection loop) or vaporization (using a vaporization roller) of small tissue chips. Tissue resection during TURP modifies significantly the shape of the prostate. The 
urologist produces a cavity inside the obstructed urethra, by resecting the adenomatous tissue until the capsule is reached.

Due to the shape of each cut during TURP, it is difficult to simulate tissue resections through geometric modifications of a tetrahedral mesh. Tetrahedral remeshing techniques are useful for tissue cutting produced by scalpels, but seem difficult to adapt for carving of small tissue chips, as it occurs during TURP. Instead, we use a simplified approach that takes advantage of the mass-spring mesh arrangement described in the previous section, based on pentahedral volume elements. Resection of tissue chips is simulated through the removal of volume elements around the resection zone, and the corresponding tissue deformation, as follows.

Let $v_{c}$ be the node of the mesh where the collision between the prostate body and the resection element of the resectoscope, occurs; let $c_{r}$ be the cutting radius of the resection element which controls the amount of tissue to be resected (typically $3 \mathrm{~mm}$ to $5 \mathrm{~mm}$ ); let $\mathbf{f}_{\mathbf{i j}}$ be the force necessary to fully compress the spring that links the nodes $\mathrm{i}$ and $\mathrm{j}$; let $\mathbf{f r}_{\mathbf{i}}$ be the resection force exerted on the node $i$ after a resection occurs (set initially to zero). Once a collision between the prostate and the resection element is detected, the closest surface node $\left(v_{c}\right)$ to the resection element is calculated. Then, the algorithm determines the list $\boldsymbol{L}$ of volume elements on the vicinity of $v_{c}$, that are under the cutting radius $c_{r}$ which must be removed from the mesh. After $\boldsymbol{L}$ is obtained, all the volume elements $e$ in $\boldsymbol{L}$ and its mechanical elements -masses $m$ in $e$, and springs $s$ adjacent to $m$, must be removed from the mesh. During a TURP the prostate deforms and the capsule may slightly collapse as the surgery progresses. In order to simulate this change of size of the prostate, the algorithm deforms the mesh as a result of the energy produced by the internal force of the springs removed. As a result of this mesh modification procedure, tissue resections are produced.

The resection algorithm progressively removes the nodes inside the vicinity of $c_{r}$ starting from the collision node $v_{c}$. The resection forces $\mathbf{f r}_{\mathbf{k}}$ acting over the $k$ nodes around $c_{r}$ after the resection, must also be progressively determined. Initially $\mathbf{f r}_{\mathbf{n}}$ is set to zero for the $n$ nodes that form the original mesh.

After a spring $i j$ is removed, the corresponding compressing force $\mathbf{f c}_{\mathbf{i j}}$ needed to move the node $i$ to the position of its $j$ neighbour is calculated. The compressing force $\mathbf{f c}_{\mathbf{i}}$ over $i$ represents the resultant force needed to move $i$ due to the contribution of $\mathbf{f c}_{\mathbf{i j}}$ for every spring $i j$ removed from the mesh. As the algorithm progresses if $i$ must also be removed from the mesh (because there are no springs connected to $i$ ), its previously computed compressing force $\mathbf{f c}_{\mathbf{i}}$ is added to the resection force $\mathbf{f r}_{\mathbf{k}}$ of all its $k$ neighbours. The algorithm continues until all the elements inside $c_{r}$ are removed from the mesh. In this way, after a resection is performed, the $\mathbf{f r}_{\mathbf{m}}$ forces acting on the resection zone around $c_{r}$, represent the resection forces acting on Eq. 1 for every node $m$ adjacent to $c_{r}$ that remains after the mesh modification. The local effect of resection is the deformation of the tissue surrounding the resection zone, as a result of the contribution of the resection forces of all the elements removed from the mesh. The global result of all resections and the corresponding local tissue deformations is the change of size of the prostatic capsule and the slight collapse of the remaining inner tissue. 


\section{Results}

The model described was implemented in $\mathrm{C}$ using the OpenGL libraries for rendering, on a Sun-Solaris workstation (with one Sparc processor @ 1 Ghz),without graphics acceleration hardware. A display rate of 8 frames/s approx. was observed for a sampling angle of $8 \mathrm{deg}$., this is below the $10 \mathrm{frames} / \mathrm{s}$ recommended as the minimum for visual realism. However significantly higher frame rates should be possible with the use of graphics acceleration hardware, since the processing time of the model alone allows for more than $12 \mathrm{frames} / \mathrm{s}$ at a sampling angle of $8 \mathrm{deg}$. In addition, for the implementation of the simulator two computers may be used, one for processing of the user interface inputs and collision detection, and the other machine for model update and display.

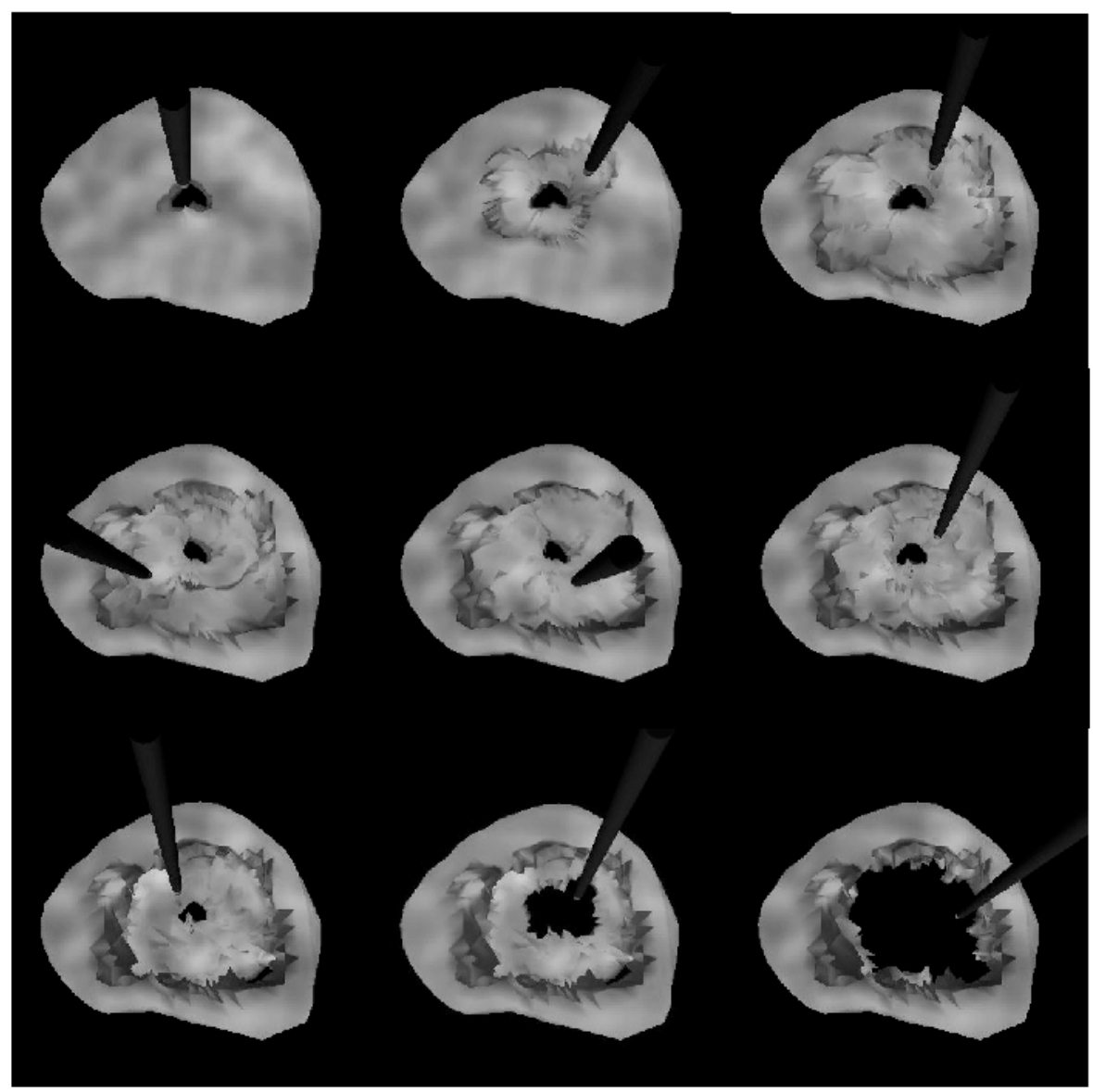

Fig. 5. Cavity produced near the urethra after some resections (from top-left to bottom-right). After every resection the tissue deforms, and after several tissue resections are performed a cavity is produced and the prostate slightly collapses 
The slides on Fig. 5 show a simulated resection of the prostate model. The figure presents a prostate with a urethra, almost completely obstructed by the tissue that has grown in excess. The slides also show the removing process of the adenoma. It can be observed the cavity produced after several tissue resections from the obstructed urethra, and the progressive change of size of the prostate capsule.

\section{Conclusions}

This paper presents a computer model of the prostate that is the basis for the development of a real-time virtual reality simulator for TURP training. Details of the construction of the geometric model of the prostate, as well as the deformation and resection simulation schemes used, are reported.

The results show that the model is well suited for resection simulation that involves tissue resection and tissue deformation. During resection simulation, when collision with the virtual resectoscope is detected, appropriate mechanical and geometrical elements from the mesh are removed and the inner tissue of the prostate is exposed. The model is able to reproduce tissue resections of different sizes, depending on the cutting radius of the resectoscope. Along with resections, the model simulates in realtime tissue deformations and the global collapse of the prostate capsule as the resection of the adenoma progresses.

The user interface for the TURP simulator is currently being developed. As a first approximation a passive mechanism with 5 degrees of freedom, instrumented with position sensors will be used to provide positional feedback only. Force feedback is not considered at this stage since the forces felt by the user during resection are small due to the vaporization of tissue. A phantom of the prostate capsule will be used to limit the resection region. The suitability of using only positional feedback will be evaluated during clinical validation of the simulator.

\section{References}

1. Yang Q., Abrams P., Donovan J., Mulligan S., Williams G.: Transurethral resection or incision of the prostate and other therapies: a survey of treatments for benign prostatic obstruction in the UK. BJU International, 84, (1999) 640-645.

2. Bro-Nielsen, M.: Finite Element Modeling in Surgery Simulation. Proceedings of the IEEE 86 No. 3 (1998) 490-503.

3. Bielser, D., Gross, M.: Interactive Simulation of surgical Cuts. Computer Graphics and Applications. Proceedings of the IEEE on The Eighth Pacific Conference, (2000) 116 442.

4. Gomes, M.P.S.F., Barret, A.R.W., Timoney, A.G., Davies, B.L.: A Computer Assisted Training/Monitoring System for TURP Structure and Design. IEEE Trans. On Information Technology in Biomed. 3 No. 4 (1999) 242-250.

5. Ballaro A., Briggs T., García-Montes F., MacDonald D., Emberton M., and Mundy A.R.: A Computer Generated Interactive Transurethral Prostatic Resection Simulator. Journal of Urology 162 (1999) 1633-1635. 
6. Nathan M.S., Mei Q., Seenivasagam K., Davies B., Wickham J.E.A. and Miller, R.A., "Comparison of prostatic volume and dimensions by transrectal and transurethral ultrasonography", British Journal of Urology, 78, pp.84-89, 1996.

7. Güdükbay, U., Özgüç, B., Tokad, Y.: A spring force formulation for elastically deformable models. Computers \& Graphics 21 No. 3 (1997) 335-346.

8. Kühnapfel, U., Cakmak H.K., MaaB, H., "Endoscopic surgery training using virtual reality and deformable tissue simulation”, Computers and Graphics, 24, pp. 671-682, 2000 